\title{
Robust formation of nanoscale magnetic skyrmions in easy-plane thin film multilayers with low damping
}

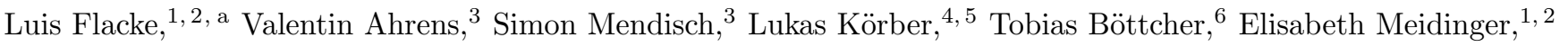 \\ Misbah Yaqoob, ${ }^{1,2}$ Manuel Müller, ${ }^{1,2}$ Lukas Liensberger, ${ }^{1,2}$ Attila Kákay, ${ }^{4}$ Markus Becherer, ${ }^{3}$ Philipp Pirro, ${ }^{6}$ \\ Matthias Althammer, ${ }^{1,2}$ Stephan Geprägs, ${ }^{1}$ Hans Huebl,,${ }^{1,2,7}$ Rudolf Gross, ${ }^{1,2,7}$ and Mathias Weiler ${ }^{1,2,6, b}$ \\ ${ }^{1}$ Walther-Meißner Institut, Bayerische Akademie der Wissenschaften, 85748 Garching, Germany \\ ${ }^{2}$ Physics Department, Technical University of Munich, 85748 Garching, Germany \\ ${ }^{3}$ Department of Electrical and Computer Engineering, \\ Technical University of Munich, 80333 Munich, Germany \\ ${ }^{4}$ Helmholtz-Zentrum Dresden-Rossendorf e.V., Institute of Ion Beam Physics and Materials Research, 01328 Dresden, Germany \\ ${ }^{5}$ Fakultät Physik, Technische Universität Dresden, 01062 Dresden, Germany \\ ${ }^{6}$ Fachbereich Physik and Landesforschungszentrum OPTIMAS, \\ Technische Universität Kaiserslautern, 67663 Kaiserslautern, Germany \\ ${ }^{7}$ Munich Center for Quantum Science and Technology (MCQST), 80799 Munich, Germany
}

(Dated: February 2021; Revised February 2021)

\begin{abstract}
We experimentally demonstrate the formation of room-temperature skyrmions with radii of about $25 \mathrm{~nm}$ in easy-plane anisotropy multilayers with interfacial Dzyaloshinskii-Moriya interaction (DMI). We detect the formation of individual magnetic skyrmions by magnetic force microscopy and find that the skyrmions are stable in out-of-plane fields up to about $200 \mathrm{mT}$. We determine the interlayer exchange coupling as well as the strength of the interfacial DMI. Additionally, we investigate the dynamic microwave spin excitations by broadband magnetic resonance spectroscopy. From the uniform Kittel mode we determine the magnetic anisotropy and low damping $\alpha_{\mathrm{G}}<0.04$. We also find clear magnetic resonance signatures in the non-uniform (skyrmion) state. Our findings demonstrate that skyrmions in easy-plane multilayers are promising for spin-dynamical applications.
\end{abstract}

Magnetic skyrmions [1] attract increasing interest for their potential use in novel devices for information storage and processing. Atomic-scale skyrmion lattices 2. and low threshold current densities for spin transfer torque movement of skyrmions were reported 3]. The room-temperature stabilization of skyrmions in entirely metallic, thin-film heterostructures [4, 5] has been identified as an important step towards real-world applications in information technologies. However, uniting all properties required for applications in a single material system is challenging. Metallic multilayer systems can be fabricated by sputter deposition techniques and allow to stabilize skyrmions at room temperature by the interface Dzyaloshinskii-Moriya interaction (iDMI) 4, 6]. This makes them promising candidates to resolve the challenges imposed by material design. For thin-film systems, an uniaxial anisotropy has been suggested to be a requirement for skyrmion formation in bulk-DMI [7] and iDMI [8] systems, which translates to perpendicular magnetic anisotropy (PMA) in thin-film heterostructures. However, both the large spin-orbit coupling materials required to induce iDMI and the very small thickness of the ferromagnet necessary for the PMA, increase damping of the magnetization dynamics due to spin pumping [9]. In turn, the high damping leads to smaller skyrmion velocities or, alternatively, requires higher current densities for fast skyrmion motion [10, 11. In addition, the PMA multilayer skyrmion systems usually is associated with a low field stability of skyrmions, as the spin textures shrink with increasing magnetic field and are annihilated by rather small external fields of $10 \mathrm{mT}-100 \mathrm{mT}$ [4] two challenges, high magnetic damping and low field stability can be overcome as discussed recently by Banerjee et al. [12. They show that switching from uniaxial to a two-dimensional (2D) easy plane anisotropy allows to significantly enhance the field robustness of the skyrmions. With this lifted PMA restriction, it is thus possible to use thicker ferromagnetic (FM) layers which still support sufficiently iDMI to create skyrmions but result in lower damping due to spin pumping.

In this work, we experimentally explore the use of a $2 \mathrm{D}$ easy plane anisotropy in magnetic multilayers for the stabilization of skyrmions. The multilayer system based on $\mathrm{Pt}, \mathrm{CoFe}$ and Ir also establishes the required iDMI and hosts skyrmions with diameter of less than $100 \mathrm{~nm}$ while showing comparatively low damping of their magnetization dynamics.

Small skyrmions in thin-film multilayers can be stabilized by the iDMI, which favors perpendicularly aligned neighboring spins $\mathbf{S}_{1,2}$ :

$$
H_{\mathrm{DMI}}=-\mathbf{D}_{12} \cdot\left(\mathbf{S}_{1} \times \mathbf{S}_{2}\right)
$$

The sign and magnitude of the iDMI vector $\mathbf{D}_{12}$ is determined by the involved materials and their stack sequence, allowing for additive DMI contributions from the two opposite interfaces of a magnetic thin film [4, 6, 13, 14.

Thin-film multilayer systems employing iDMI for skyrmion formation typically rely on PMA. This is reflected in the condition for the effective anisotropy $K_{\text {eff }}=$ $K_{\mathrm{u}}-\mu_{0} M_{\mathrm{s}}^{2} / 2>0$, which omits higher order spin orbit 
a)
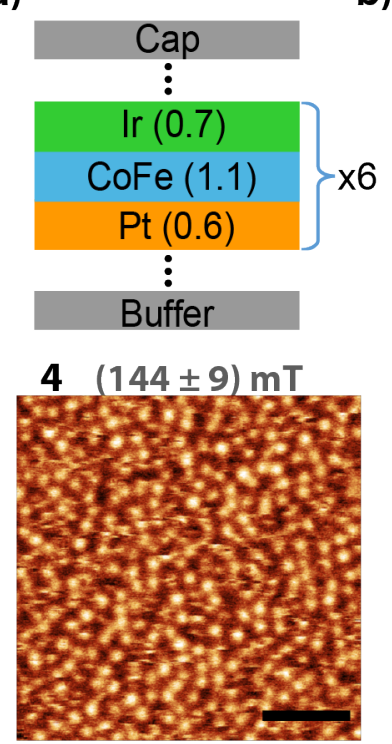

b) $1(309 \pm 15) \mathrm{mT}$

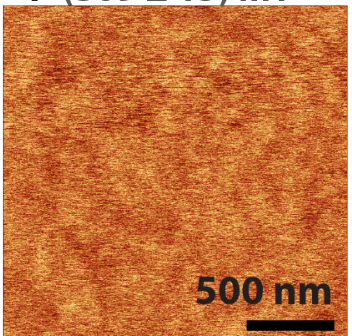

$5(113 \pm 7) \mathrm{mT}$

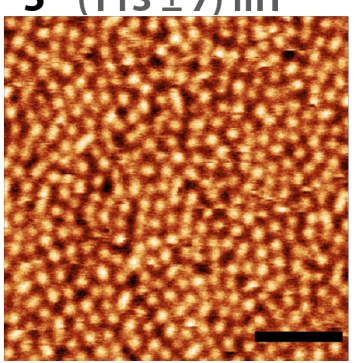

$2(186 \pm 10) \mathrm{mT}$

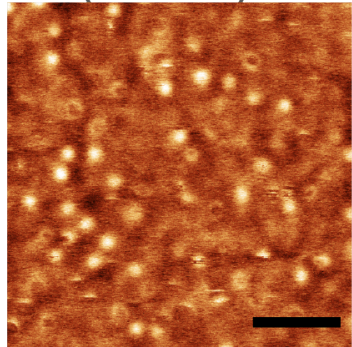

$6 \quad(97 \pm 6) \mathrm{mT}$

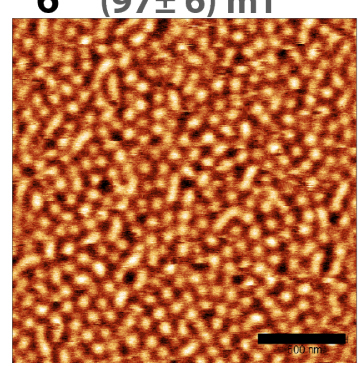

$3(171 \pm 10) \mathrm{mT}$
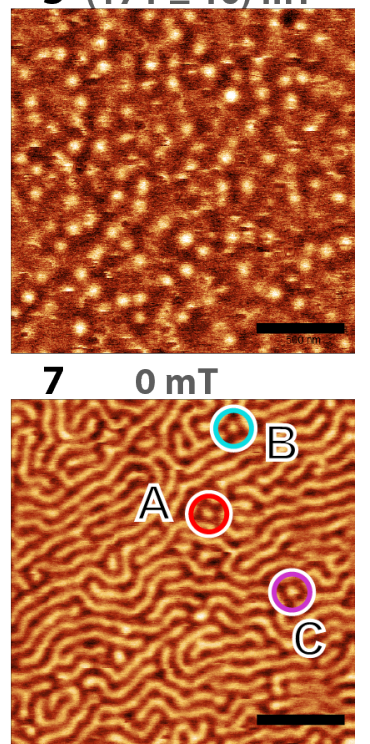

FIG. 1. a) Sketch of the multilayer system, with a sixfold repetition of the Pt/CoFe/Ir trilayer (numbers give the film thickness in $\mathrm{nm}$ ). b) MFM images recorded with OOP magnetic fields, field values are indicated at the top of each frame. With decreasing field, the magnetic texture shows transitions from a saturated ferromagnetic state (1), to isolated skyrmions $(2,3)$, to a dense, skyrmion arrangement $(4-6)$ and eventually forms a maze state at zero field (7). Individual skyrmions between the spin-spirals can still be found at zero field. The three highlighted skyrmions (marked with three colored circles in (7)) are analyzed further in Fig. 2]a). Scalebar in all images corresponds to $500 \mathrm{~nm}$.

coupling terms and dipolar contributions [7, 8]. Here, $K_{\mathrm{u}}>0$ represents an anisotropy with easy axis along the film normal (out-of-plane, OOP), and the second term describes the shape anisotropy of the thin film field with the vacuum permeability $\mu_{0}$ and the saturation magnetization $M_{\mathrm{s}}$. Recent theoretical calculations however also predict the existence of skyrmions with increased field stability within the $K_{\text {eff }}<0$ regime [12, 15, 17]. Not requiring PMA allows to use thicker ferromagnetic (FM) layers with reduced spin pumping damping contributions, while iDMI is still sufficiently large to enable skyrmion formation.

We use sputter deposited heterostructues composed of $\left[\mathrm{Pt}(0.6) / \mathrm{Co}_{25} \mathrm{Fe}_{75}(1.1) / \operatorname{Ir}(0.7)\right]_{6}$ multilayers (numbers give the layer thickness in nanometers), as sketched in Fig. 1 a) to reveal the abovementioned properties and benefits. Additionally, we fabricated a sample series with varying Ir thickness in order to determine the interlayer RKKY coupling. In the following, we denote the specific $\mathrm{Co}_{25} \mathrm{Fe}_{75}$ alloy simply as CoFe. The details of the sample fabrications are discussed in the supplementary information (SI) [18].

In order to observe of the formation of skyrmions in our multilayers, we use magnetic force microscopy (MFM). Fig. 1 b) presents $2 \mu \mathrm{m} \times 2 \mu \mathrm{m}$ MFM phase contrast images recorded with external magnetic fields $0 \mathrm{mT} \leq \mu_{0} H_{\text {ext }} \leq 309 \mathrm{mT}$ applied in the OOP direction. In the following, we discuss the individual MFM images shown in Fig. 1 b). MFM image (1) shows van- ishing contrast, as the sample is magnetically saturated, meaning that all magnetic moments are aligned parallel to the applied magnetic field. By reducing $\mu_{0} H_{\text {ext }}$ below the saturation magnetic field, small individual dots with an apparent diameter below $100 \mathrm{~nm}$ start to emerge (2), which we attribute to skyrmion formation. A fixed chirality of the skyrmions is expected, as we determine a high interface DMI value of $D_{\mathrm{int}} \approx 1.86 \mathrm{~mJ} / \mathrm{m}^{2}$ with BLS measurements [19] on reference samples (see SI [18]). This value agrees well with DMI strengths reported for superlattices with similar materials [13]. The density of skyrmions increases further on reducing the magnetic field magnitude until a dense, unordered arrangement is formed $(3-6)$. Even lower magnetic fields eventually lead to the formation of a labyrinthine state at remanence, shown in image (7). However, obviously individual skyrmions maintain stabilized even at $H_{\text {ext }}=0$, as indicated by the three circles. The multilayer thus hosts skyrmions for $0 \leq \mu_{0} H_{\text {ext }} \leq 190 \mathrm{mT}$. This field range for skyrmion formation is roughly twice the range reported for to $K_{\text {eff }} \geq 0$ systems [4, 6, 13, 20, 21].

In Fig. 2 we take a closer look at the magnetic field dependent skyrmion size distribution to elucidate the qualitatively different behavior compared to PMA systems [4, 5, 22, . Fig. 20) depicts the azimuthally averaged radial profile of the magnetic contrast of the three highlighted skyrmions in image (7) of Fig.1 b) vs. the radial distance from their center position. Using a Gaussian fit, we extract the skyrmion diameter $d$ as the full-width-half- 

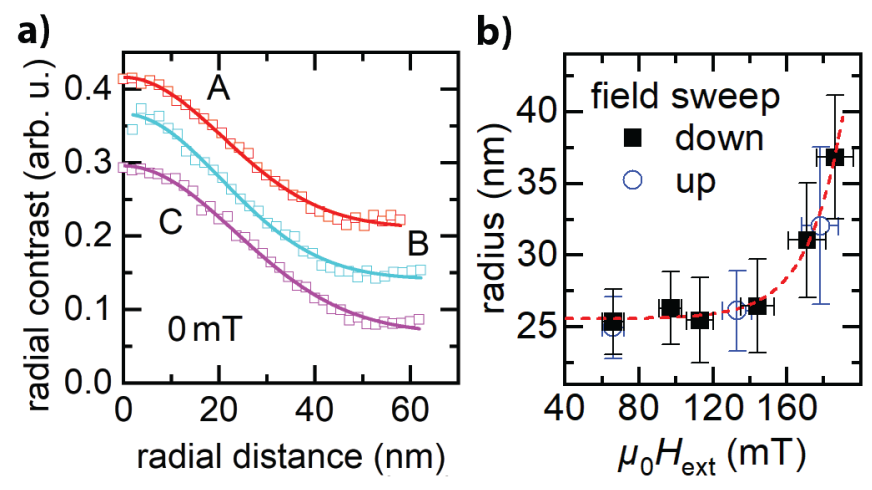

FIG. 2. a) Azimuthally averaged MFM phase contrast of the skyrmions highlighted in Fig. 1 (offset is used for clarity). The radial profile is fitted with a Gaussian function, from which the radius $r=(25.4 \pm 1.6) \mathrm{nm}$ is found at $H_{\text {ext }}=$ 0 . b) Apparent radius vs. applied magnetic field in OOP direction. The black squares indicate the average value taken from 28-115 skyrmions from images $(2-6)$ of Fig. 1 b) for a field down sweep starting at saturation, whereas, the blue circles indicate skyrmion sizes determined from an up sweep starting at $(66 \pm 6) \mathrm{mT}$. The $y$-error represents the standard deviation of the size distribution. The $x$-error bar indicates the uncertainty of the field calibration. The red dotted line is a guide to the eye.

maximum of the MFM signal. We define the apparent radius as $r=d / 2$. At $\mu_{0} H_{\text {ext }}=0 \mathrm{mT}$, the three extracted radii are about $25 \mathrm{~nm}$. For larger $\mu_{0} H_{\text {ext }}$ we extracted the radial profile of $28-115$ skyrmions from graphs $(2-6)$ and plot the average radius and its standard deviation vs. the applied field. For $\mu_{0} H_{\text {ext }}<150 \mathrm{mT}, r$ remains unchanged within experimental uncertainty, while $r$ diverges at the transition between the skyrmion state and the ferromagnetic alignment. This behavior is in contrast to the typically observed reduction of $r$ with $H_{\text {ext }}$ observed for skyrmions in PMA-based multilayers 4, 5, 13. We additionally verified experimentally that the skyrmion formation shown in Fig. 1 is nonhysteretic within experimental uncertainty, i.e., the same skyrmion size is obtained for given $\mu_{0} H_{\text {ext }}$, regardless of field history (see SI 18]). This indicates a strong influence of the iDMI on the skyrmion formation in our samples.

We note that a qualitatively similar radius increase can be found in antiferromagnetically coupled skyrmion bilayer systems [23]. However, no antiferromagnetic (AFM) coupling was found in our sample. By employing anomalous Hall effect (AHE) measurements to extract the saturation field $\mu_{0} H_{\mathrm{S}}$ (see SI [18]) and varying the Ir spacer thickness as shown in Fig. 3a) the AFM and FM RKKY coupling regions can be separated. Ir has been shown to induce strong AFM coupling between magnetic layers in the ultra-thin limit [24, 25. As the coupling strength varies significantly with Ir thickness, so does the required field to overcome the AFM order and obtain saturation. Within the FM regime, $\mu_{0} H_{\mathrm{S}}$ stays constant 26]. The sample studied by MFM has an Ir thickness $t_{\mathrm{Ir}}=7 \AA$ and is thus FM coupled. This rules out AFM coupling as the origin of the observed independence of skyrmion size on external magnetic field magnitude.
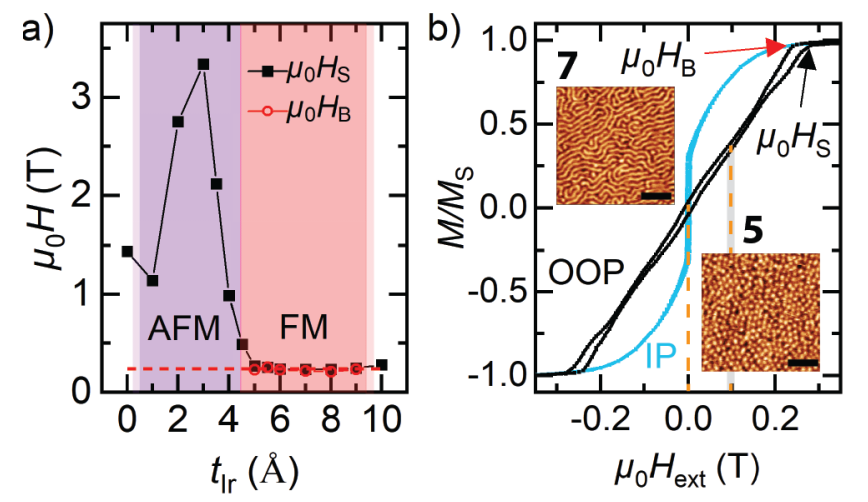

FIG. 3. a) Saturation fields $\mu_{0} H_{\mathrm{S}}$ of samples with varying Ir thickness $t_{\mathrm{Ir}}$ are determined by anomalous Hall effect measurements. In the AFM RKKY coupling regime, $H_{\mathrm{S}}$ correlates with the strength of exchange coupling. Within the FM regime, $H_{\mathrm{S}}$ is independent of coupling strength, and small pockets in the hysteresis curves allow for a qualitative separation of the regimes with AFM and FM coupling. Corresponding samples show a characteristic field $\mu_{0} H_{\mathrm{B}}<\mu_{0} H_{\mathrm{S}}$ below which saturation starts to break down. b) The hysteresis curves from the sample discussed in Fig. 1 are recorded by SQUID magnetometry in IP and OOP direction. The insets depict MFM images at the indicated fields (vertical dotted lines) along the descending OOP branch. The IP graph exhibits an easy-plane switching loop with a coercivity of less than $4 \mathrm{mT}$. The decrease of magnetic moment for $\left|\mu_{0} H_{\text {ext }}\right|<200 \mathrm{mT}$ is attributed to domain formation due to iDMI. Scalebar in all images corresponds to $500 \mathrm{~nm}$.

Our observation is in accordance with the theoretical work by Banerjee et al. who predicted a stable skyrmion size for a large field range for easy-plane anisotropy thin films. The contribution of the easy-plane "compass" anisotropy to the free energy stemming from higher order spin-orbit coupling terms 12 can possibly explain why we observe an almost magnetic field independent skyrmion size. This is further supported by the observation of a qualitatively different skyrmion size evolution in our micromagnetic simulations that do not include anisotropies caused by higher order spin-orbit coupling terms (see SI [18])

For our system, the easy plane anisotropy is verified by superconducting quantum interference device (SQUID) magnetometry. We show the in-plane (IP) and the OOP magnetization curves in Fig. [3 b). The shape of the OOP $M$ vs. $H_{\text {ext }}$ loop is characteristic for samples with a maze-state formation, where skyrmions are often found [6, 13, 24]. Due to domain formation, also PMA systems involving labyrinthine arrangement can exhibit 


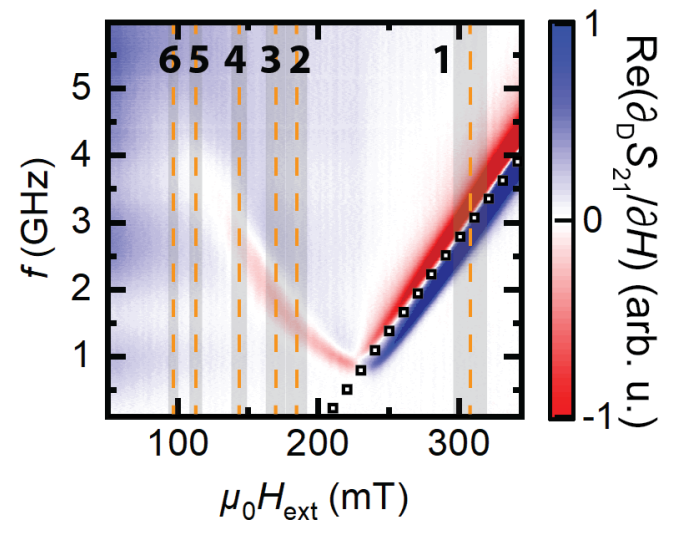

FIG. 4. The colorplot shows the real part of the background corrected FMR signal. For $\mu_{0} H_{\text {ext }}>240 \mathrm{mT}$ applied along the OOP direction we see the characteristic FM response of the CoFe-multilayer. The black squares indicate the micromagnetic simulation results (see SI [18]). Below the critical field we see an additional dynamic response, which we attribute to the skyrmion background. Dashed lines and the gray area indicate the field and its uncertainty, respectively, at which the correspondingly numbered MFM graphs in Fig. 1 were recorded. The Gilbert damping parameter is $\alpha_{\mathrm{G}}=(37 \pm 1) \times 10^{-3}($ see SI 18$)$.

a similar hysteresis curve [13, 24]. We verify the easy plane anisotropy by performing IP SQUID magnetometry measurements. The IP behavior qualitatively differs from the OOP curve and also from $K_{\text {eff }} \geq 0$ systems, where the IP curve shows hard-axis behavior [13, 20]. We observe an easy-plane switching loop with a coercivity of less than $4 \mathrm{mT}$. The gradual decrease of magnetization for $\mu_{0} H_{\text {ext }} \leq 200 \mathrm{mT}$ is attributed to domain formation. The formation of a maze state at remanence despite easyplane anisotropy is attributed to the strong iDMI [27.

We have so far demonstrated that stable skyrmions can form in easy-plane anisotropy thin films with DMI. We now turn to the dynamic magnetic properties of such thin film multilayers. To this end, we determine the dynamic microwave response of the sample by placing it on a coplanar waveguide (CPW) and recording the microwave transmission $S_{21}$ through the CPW with a vector network analyzer (VNA) 28, 29. The frequency dependent background is removed by calculating the normalized field-derivative [30] $\partial_{\mathrm{D}} S_{21} / \partial H$ and $\operatorname{Re}\left(\partial_{\mathrm{D}} S_{21} / \partial H\right)$ is shown in Fig. 4 .

For $\mu_{0} H_{\text {ext }} \gtrsim 240 \mathrm{mT}$, we observe the ferromagnetic resonance (FMR) of the fully aligned CoFe magnetization, which follows the known OOP Kittel equation for thin films [31]:

$$
2 \pi f_{\mathrm{res}}=\mu_{0} \gamma\left(H_{\mathrm{ext}}-M_{\mathrm{eff}}\right) .
$$

Here, $\gamma$ is the gyromagnetic ratio, $H_{\text {ext }}$ is the applied magnetic field, and $M_{\mathrm{eff}}=M_{\mathrm{s}}-H_{\mathrm{k}}$ with the perpendicular anisotropy field $H_{\mathrm{k}}=2 K_{\mathrm{u}} /\left(\mu_{0} M_{\mathrm{S}}\right)$. We fit the data in Fig. 4 to extract resonance fields and linewidths as detailed in the SI [18. We determine $\mu_{0} M_{\text {eff }}=(201.8 \pm$ $0.1) \mathrm{mT}$, again confirming the easy plane anisotropy. We used the determined parameters, as input variables for micromagnetic simulations and were able to qualitatively reproduce the hysteresis curves as well as the formation of chiral spin textures within the $\mu_{0} M_{\text {eff }}>0$ system (see SI [18). From fitting the linewidth of the signal for a broad frequency range we extract the Gilbert damping parameter and obtain $\alpha_{\mathrm{G}}=0.037$ (see SI [18]). This value is almost an order of magnitude lower than those reported on skyrmion host multilayers of $\mathrm{Pt} / \mathrm{CoFeB} / \mathrm{MgO}$ $(\alpha=0.5)[32$ and $\mathrm{Ir} / \mathrm{Fe} / \mathrm{Co} / \mathrm{Pt}(\alpha=0.1)$ [13. Another common FM material used for skyrmion multilayers is Co, where $\alpha \approx 0.3$ is determined for layer thicknesses $t<1 \mathrm{~nm}$ sandwiched between Pt layers 33. As pointed out by Fert et al., such a decrease in magnetic damping is expected to result in substantial improvement for skyrmion motion [11].

For $\mu_{0} H_{\text {ext }}<240 \mathrm{mT}, f_{\text {res }}$ vs. $H_{\text {ext }}$ shows a qualitatively different behavior with $f_{\text {res }}$ increasing for decreasing $\left|H_{\text {ext }}\right|$. This is attributed to a rotation of $M$ towards the film plane and the formation of a non-uniform magnetic texture in accordance with our MFM and SQUID magnetometry data. We attribute the resonance observed for $\mu_{0} H_{\text {ext }}<240 \mathrm{mT}$ to the dynamic precession of the magnetic moments in the quasi-uniform background (see Fig. 1) and not to spin dynamics within the skyrmions. This is in accordance with the vanishing amplitude of the resonance towards $H_{\text {ext }}=0$, where no uniform background remains. Our observation is qualitatively different to that made by Montoya et al. [34, where various resonances of dipolar skyrmions in DMIless Fe/Gd superlattices were observed. Even though the magnetic resonance data look similar, Montoya et al. observed a phase transition of a field polarized state directly to a phase with coexisting stripes and skyrmions. Our MFM data, however, reveal a smooth transition from parallel alignment, over individual skyrmions, to a dense skyrmion arrangement and eventually a maze state formation. Our results are also qualitatively different from a study of PMA iDMI thin-films with magnetic skyrmions [35, where magnetic resonance is also observed in a phase with coexisting stripes and skyrmions.

We explicitly confirm a larger magnetic field range with skyrmion existence than comparable PMA systems 4, 6, 13, 20, 21] by MFM. Our measurements show a constant skyrmion size over a wide range of external magnetic fields $(0 \mathrm{mT}$ to $\sim 150 \mathrm{mT})$ as predicted by Banerjee et al. [12. This size evolution opposes the typical decrease of skyrmion size with increasing magnetic field due to Zeeman energy contributions 4. We explicitly rule out antiferromagnetic coupling between layers as an explanation for the unusual size dependence by varying the spacer thickness. The easy-plane anisotropy in our films is verified by (SQUID) magnetometry and broad- 
band magnetic resonance. Microwave spectroscopy experiments reveal a happily low damping within the system, making it promising for more detailed studies of spin dynamics. Compared to other experimentally realized easy-plane skyrmion systems like polar magnets 36, bulk- and interface DMI thin films [37, 38, as well as DMI-less Fe/Gd superlattices [39], the investigated multilayer system uniquely provides room-temperature nanoscale skyrmions in a low damping host material for a large field range. The significant reduction of damping can result in faster skyrmion motion [11]. The high stability of the skyrmion size over a broad range of external magnetic fields may be also beneficial for devices.

Acknowledgments. - We acknowledge financial support by the Deutsche Forschungsgemeinschaft (DFG, German Research Foundation) via WE5386/4-1, WE5386/51 and Germany's Excellence Strategy EXC-2111390814868 .

a luis.flacke@wmi.badw.de

b weiler@physik.uni-kl.de

[1] S. Muhlbauer, B. Binz, F. Jonietz, C. Pfleiderer, A. Rosch, A. Neubauer, R. Georgii, and P. Boni, Skyrmion Lattice in a Chiral Magnet, Science 323, 915 (2009)

[2] S. Heinze, K. Von Bergmann, M. Menzel, J. Brede, A. Kubetzka, R. Wiesendanger, G. Bihlmayer, and S. Blügel, Spontaneous atomic-scale magnetic skyrmion lattice in two dimensions, Nature Physics 7, 713 (2011).

[3] F. Jonietz, S. Muhlbauer, C. Pfleiderer, A. Neubauer, W. Munzer, A. Bauer, T. Adams, R. Georgii, P. Boni, R. A. Duine, K. Everschor, M. Garst, and A. Rosch, Spin Transfer Torques in MnSi at Ultralow Current Densities, Science 330, 1648 (2010)

[4] C. Moreau-Luchaire, C. Moutafis, N. Reyren, J. Sampaio, C. A. F. Vaz, N. Van Horne, K. Bouzehouane, K. Garcia, C. Deranlot, P. Warnicke, P. Wohlhüter, J.-M. George, M. Weigand, J. Raabe, V. Cros, and A. Fert, Additive interfacial chiral interaction in multilayers for stabilization of small individual skyrmions at room temperature, Nature Nanotechnology 11, 444 (2016).

[5] O. Boulle, J. Vogel, H. Yang, S. Pizzini, D. de Souza Chaves, A. Locatelli, T. O. Menteş, A. Sala, L. D. BudaPrejbeanu, O. Klein, M. Belmeguenai, Y. Roussigné, A. Stashkevich, S. M. Chérif, L. Aballe, M. Foerster, M. Chshiev, S. Auffret, I. M. Miron, and G. Gaudin, Room-temperature chiral magnetic skyrmions in ultrathin magnetic nanostructures, Nature Nanotechnology 11, 449 (2016)

[6] S. Woo, K. Litzius, B. Krüger, M. Y. Im, L. Caretta, K. Richter, M. Mann, A. Krone, R. M. Reeve, M. Weigand, P. Agrawal, I. Lemesh, M. A. Mawass, P. Fischer, M. Kläui, and G. S. Beach, Observation of room-temperature magnetic skyrmions and their currentdriven dynamics in ultrathin metallic ferromagnets, $\mathrm{Na}-$ ture Materials 15, 501 (2016)

[7] M. N. Wilson, A. B. Butenko, A. N. Bogdanov, and T. L. Monchesky, Chiral skyrmions in cubic helimagnet films:
The role of uniaxial anisotropy, Physical Review B 89, 094411 (2014)

[8] X. S. Wang, H. Y. Yuan, and X. R. Wang, A theory on skyrmion size, Communications Physics 1, 31 (2018)

[9] Y. Tserkovnyak, A. Brataas, and G. E. W. Bauer, Enhanced Gilbert Damping in Thin Ferromagnetic Films, Physical Review Letters 88, 117601 (2002).

[10] J. Iwasaki, M. Mochizuki, and N. Nagaosa, Currentinduced skyrmion dynamics in constricted geometries, Nature Nanotechnology 8, 742 (2013)

[11] A. Fert, N. Reyren, and V. Cros, Magnetic skyrmions: advances in physics and potential applications, Nature Reviews Materials 2, 17031 (2017)

[12] S. Banerjee, J. Rowland, O. Erten, and M. Randeria, Enhanced Stability of Skyrmions in Two-Dimensional Chiral Magnets with Rashba Spin-Orbit Coupling, Physical Review X 4, 031045 (2014).

[13] A. Soumyanarayanan, M. Raju, A. L. Gonzalez Oyarce, A. K. C. Tan, M.-Y. Im, A. P. Petrović, P. Ho, K. H. Khoo, M. Tran, C. K. Gan, F. Ernult, and C. Panagopoulos, Tunable room-temperature magnetic skyrmions in $\mathrm{Ir} / \mathrm{Fe} / \mathrm{Co} / \mathrm{Pt}$ multilayers, Nature Materials 16, $898(2017)$.

[14] H. Yang, O. Boulle, V. Cros, A. Fert, and M. Chshiev, Controlling Dzyaloshinskii-Moriya Interaction via Chirality Dependent Atomic-Layer Stacking, Insulator Capping and Electric Field, Scientific Reports 8, 12356 (2018)

[15] S.-Z. Lin, A. Saxena, and C. D. Batista, Skyrmion fractionalization and merons in chiral magnets with easyplane anisotropy, Physical Review B 91, 224407 (2015).

[16] J. Rowland, S. Banerjee, and M. Randeria, Skyrmions in chiral magnets with Rashba and Dresselhaus spin-orbit coupling, Physical Review B 93, 020404 (2016).

[17] M. Vousden, M. Albert, M. Beg, M.-A. Bisotti, R. Carey, D. Chernyshenko, D. Cortés-Ortuño, W. Wang, O. Hovorka, C. H. Marrows, and H. Fangohr, Skyrmions in thin films with easy-plane magnetocrystalline anisotropy, Applied Physics Letters 108, 132406 (2016).

[18] See Supplemental Material [url], which includes additional Refs. [40 44] for details about fabrication, supporting MFM, BLS, AHE and FMR measurements and micromagnetic simulations..

[19] H. T. Nembach, J. M. Shaw, M. Weiler, E. Jué, and T. J. Silva, Linear relation between Heisenberg exchange and interfacial Dzyaloshinskii-Moriya interaction in metal films, Nature Physics 11, 825 (2015).

[20] W. Legrand, D. Maccariello, F. Ajejas, S. Collin, A. Vecchiola, K. Bouzehouane, N. Reyren, V. Cros, and A. Fert, Room-temperature stabilization of antiferromagnetic skyrmions in synthetic antiferromagnets, Nature Materials 19, 34 (2020)

[21] K. Zeissler, M. Mruczkiewicz, S. Finizio, J. Raabe, P. M. Shepley, A. V. Sadovnikov, S. A. Nikitov, K. Fallon, S. McFadzean, S. McVitie, T. A. Moore, G. Burnell, and C. H. Marrows, Pinning and hysteresis in the field dependent diameter evolution of skyrmions in $\mathrm{Pt} / \mathrm{Co} / \mathrm{Ir}$ superlattice stacks, Scientific Reports 7, 15125 (2017).

[22] N. Romming, A. Kubetzka, C. Hanneken, K. von Bergmann, and R. Wiesendanger, Field-Dependent Size and Shape of Single Magnetic Skyrmions, Physical Review Letters 114, 177203 (2015)

[23] M. N. Potkina, I. S. Lobanov, H. Jónsson, and V. M. Uzdin, Skyrmions in antiferromagnets: Thermal stability 
and the effect of external field and impurities, Journal of Applied Physics 127, 213906 (2020)

[24] O. Hellwig, A. Berger, J. B. Kortright, and E. E. Fullerton, Domain structure and magnetization reversal of antiferromagnetically coupled perpendicular anisotropy films, Journal of Magnetism and Magnetic Materials 319, $13(2007)$

[25] M. S. Gabor, T. Petrisor, R. B. Mos, M. Nasui, C. Tiusan, and T. Petrisor, Interlayer exchange coupling in perpendicularly magnetized $\mathrm{Pt} / \mathrm{Co} / \mathrm{Ir} / \mathrm{Co} / \mathrm{Pt}$ structures, Journal of Physics D: Applied Physics 50, 465004 (2017)

[26] S. Karayev, P. D. Murray, D. Khadka, T. R. Thapaliya, K. Liu, and S. X. Huang, Interlayer exchange coupling in $\mathrm{Pt} / \mathrm{Co} / \mathrm{Ru}$ and $\mathrm{Pt} / \mathrm{Co} / \mathrm{Ir}$ superlattices, Physical Review Materials 3, 041401 (2019)

[27] K.-W. Moon, J. Yoon, C. Kim, and C. Hwang, Existence of in-Plane Magnetic Skyrmion and its Motion under Current Flow, Physical Review Applied 12, 064054 (2019)

[28] S. S. Kalarickal, P. Krivosik, M. Wu, C. E. Patton, M. L. Schneider, P. Kabos, T. J. Silva, and J. P. Nibarger, Ferromagnetic resonance linewidth in metallic thin films: Comparison of measurement methods, Journal of Applied Physics 99, 093909 (2006).

[29] M. Weiler, A. Aqeel, M. Mostovoy, A. Leonov, S. Geprägs, R. Gross, H. Huebl, T. T. Palstra, and S. T. Goennenwein, Helimagnon Resonances in an Intrinsic Chiral Magnonic Crystal, Physical Review Letters 119, 237204 (2017)

[30] H. Maier-Flaig, S. T. Goennenwein, R. Ohshima, M. Shiraishi, R. Gross, H. Huebl, and M. Weiler, Note: Derivative divide, a method for the analysis of broadband ferromagnetic resonance in the frequency domain, Review of Scientific Instruments 89, 89 (2018)

[31] C. Kittel, On the theory of ferromagnetic resonance absorption, Physical Review 73, 155 (1948)

[32] K. Litzius, I. Lemesh, B. Krüger, P. Bassirian, L. Caretta, K. Richter, F. Büttner, K. Sato, O. A. Tretiakov, J. Förster, R. M. Reeve, M. Weigand, I. Bykova, H. Stoll, G. Schütz, G. S. D. Beach, and M. Kläui, Skyrmion Hall effect revealed by direct time-resolved X-ray microscopy, Nature Physics 13, 170 (2017).

[33] P. J. Metaxas, J. P. Jamet, A. Mougin, M. Cormier, J. Ferré, V. Baltz, B. Rodmacq, B. Dieny, and R. L. Stamps, Creep and flow regimes of magnetic domain-wall motion in ultrathin $\mathrm{Pt} / \mathrm{Co} / \mathrm{Pt}$ films with perpendicular anisotropy, Physical Review Letters 99, 1 (2007).

[34] S. A. Montoya, S. Couture, J. J. Chess, J. C. T. Lee, N. Kent, M.-Y. Im, S. D. Kevan, P. Fischer, B. J. McMorran, S. Roy, V. Lomakin, and E. E. Fullerton, Reso- nant properties of dipole skyrmions in amorphous Fe/Gd multilayers, Physical Review B 95, 224405 (2017).

[35] B. Satywali, F. Ma, S. He, M. Raju, V. P. Kravchuk, M. Garst, A. Soumyanarayanan, and C. Panagopoulos, Gyrotropic resonance of individual Néel skyrmions in Ir/Fe/Co/Pt multilayers, (2018), arXiv:1802.03979

[36] S. Bordács, A. Butykai, B. G. Szigeti, J. S. White, R. Cubitt, A. O. Leonov, S. Widmann, D. Ehlers, H. A. Von Nidda, V. Tsurkan, A. Loidl, and I. Kézsmárki, Equilibrium skyrmion lattice ground state in a polar easy-plane magnet, Scientific Reports 7, 1 (2017).

[37] A. S. Ahmed, J. Rowland, B. D. Esser, S. R. Dunsiger, D. W. McComb, M. Randeria, and R. K. Kawakami, Chiral bobbers and skyrmions in epitaxial FeGe/Si(111) films, Physical Review Materials 2, 041401 (2018)

[38] M. He, G. Li, Z. Zhu, Y. Zhang, L. Peng, R. Li, J. Li, H. Wei, T. Zhao, X.-G. Zhang, S. Wang, S.-Z. Lin, L. Gu, G. Yu, J. W. Cai, and B.-g. Shen, Evolution of topological skyrmions across the spin reorientation transition in Pt/Co/Ta multilayers, Physical Review B 97, 174419 (2018)

[39] S. A. Montoya, S. Couture, J. J. Chess, J. C. Lee, N. Kent, D. Henze, S. K. Sinha, M. Y. Im, S. D. Kevan, P. Fischer, B. J. McMorran, V. Lomakin, S. Roy, and E. E. Fullerton, Tailoring magnetic energies to form dipole skyrmions and skyrmion lattices, Physical Review B 95, 1 (2017).

[40] J. Agustsson, U. Arnalds, A. Ingason, K. Gylfason, K. Johnsen, S. Olafsson, and J. Gudmundsson, Growth, coalescence, and electrical resistivity of thin $\mathrm{Pt}$ films grown by dc magnetron sputtering on $\mathrm{SiO} 2$, Applied Surface Science 254, 7356 (2008)

[41] G. Woltersdorf, F. Hoffmann, H. G. Bauer, and C. H. Back, Magnetic homogeneity of the dynamic properties of ( $\mathrm{Ga}, \mathrm{Mn})$ As films from the submicrometer to millimeter length scale, Physical Review B 87, 054422 (2013)

[42] R. D. McMichael, D. J. Twisselmann, and A. Kunz, Localized Ferromagnetic Resonance in Inhomogeneous Thin Films, Physical Review Letters 90, 4 (2003)

[43] A. Vansteenkiste, J. Leliaert, M. Dvornik, M. Helsen, F. Garcia-Sanchez, and B. Van Waeyenberge, The design and verification of MuMax3, AIP Advances 4, 107133 (2014).

[44] H. S. Körner, M. A. W. Schoen, T. Mayer, M. M. Decker, J. Stigloher, T. Weindler, T. N. G. Meier, M. Kronseder, and C. H. Back, Magnetic damping in poly-crystalline Co 25 Fe 75 : Ferromagnetic resonance vs. spin wave propagation experiments, Applied Physics Letters 111, 132406 (2017). 